\title{
CARACTERÍSTICAS FISIOLÓGICAS, CONTENIDO DE ACEITE Y PROTEÍNA EN GENOTIPOS DE SOYA, EVALUADAS EN DIFERENTES SITIOS Y ÉPOCAS DE COSECHA, BRASIL
}

\author{
Physiologic characteristics, oil and protein content in soybean genotypes across site \\ and harvest period, Brazil
}

\author{
Andréia Minuzzi ${ }^{1}$, Freddy Mora ${ }^{1 *}$, Marco Antônio Sedrez Rangel ${ }^{2}$, Alessandro De Lucca e \\ Braccini $^{1}$, y Carlos Alberto Scapim ${ }^{1}$
}

\section{A B S T R A C T}

The objective of this study was to evaluate physiologic characteristics and oil and protein content in four soybean (Glycine max (L.) Merr.) cultivars (BRS 133, BRS 206, BRS 239 and CD 202) which were harvested in four different periods $\left(\mathrm{R}_{7}\right.$ stage, 7,14 and 21 days after the first harvest stage) and produced over the growing season 2004-2005 in two sites of the Mato Grosso do Sul State, Brazil, Sidrolândia ( $21^{\circ} 00^{\prime}$ S lat, $55^{\circ} 01^{\prime} \mathrm{W}$ long), and Dourados $\left(22^{\circ} 11^{\prime}\right.$ 'S lat, $54^{\circ} 55^{\prime} \mathrm{W}$ long). A randomized complete block design was used in a split-plot experiment with three replicates. Seeds were evaluated by means of a germination test, accelerated aging and seedling emergence on sand. Oil content and protein in seed and soybean meal were also analyzed. BRS 133 and BRS 206 cultivars showed higher values of seed-protein, 35.67 and $35.0 \%$, respectively, which also evidenced higher soybean meal protein content, 43.04 and $46.83 \%$, respectively. The highest oil content was found in cv. CD 202, with a mean value of $22.63 \%$. Germination and seed vigor potentials were significantly affected by harvest periods, which presented a significant reduction when soybean harvest was delayed, such characteristics could affect the plant potential for fast growth and adequate develop of soybean plants.

Key words: Glycine max, germination, delayed harvest, cultivars.

\section{R E S U M E N}

El objetivo del presente estudio fue evaluar características fisiológicas, contenido de aceite y de proteínas en las semillas de cuatro cultivares de soya (Glycine $\max$ (L.) Merr.) (BRS 133, BRS 206, BRS 239 y CD 202), colectadas en cuatro épocas (estado $R_{7}, 7$, 14 y 21 días después de la primera época) y producidas en dos sitios, Sidrolândia $\left(21^{\circ} 00^{\prime}\right.$ lat. Sur, $55^{\circ} 01^{\prime}$ long. Oeste), y Dourados ( $22^{\circ} 11^{\prime}$ lat. Sur, $54^{\circ} 55^{\prime}$ long. Oeste) del Estado de Mato Grosso do Sul, Brasil, en la temporada agrícola 2004-2005. Se utilizó un diseño experimental de bloques completos al azar con tres repeticiones y con tratamientos en parcelas subdivididas. Las semillas fueron evaluadas a través de pruebas de germinación, envejecimiento acelerado y emergencia de las plantas en substrato de arena. Se determinaron los contenidos de aceite y proteínas en las semillas y en el afrecho de soya. Los cvs. BRS 133 y BRS 206 presentaron los mayores contenidos de proteínas, 35,67 y $35,0 \%$, respectivamente, los cuales también mostraron los mayores valores de proteína en el afrecho de soya, 43,04 y $46,83 \%$, respectivamente. El mayor valor en el contenido de aceite se encontró en el cv. CD 202, con un valor medio de $22,63 \%$. A medida que la época de cosecha fue retardada, se observó una reducción significativa en el potencial germinativo y en el vigor de las semillas, características que podrían determinar el potencial para un crecimiento rápido y un desarrollo adecuado de la planta de soya.

Palabras clave: Glycine max, germinación, atraso de cosecha, cultivares.

\footnotetext{
${ }^{1}$ Universidade Estadual de Maringá, Departamento de Agronomía, Av. Colombo 5790, Bloco 32, CEP: 87020-900, Maringá, Paraná, Brasil. E-mail:morapoblete@gmail.com; andminuzi@zipmail.com.br; albraccini@uem.br; cascapim@uem.br *Autor para correspondencia. ${ }^{2}$ Empresa de Pesquisa Agropecuária, EMBRAPA Agropecuária Oeste, Caixa Postal 661, CEP 79804-970, Dourados, Mato Grosso do Sul, Brasil. E-mail: rangel@cpao.embrapa.br

Recibido: 6 de octubre de 2006. Aceptado: 12 de enero de 2007.
} 


\section{INTRODUCCIÓN}

La soya, tanto en forma de grano como en sus subproductos, posee un alto contenido proteico, el cual es un factor importante para la alimentación humana y animal. Su cultivo tiene gran importancia económica y social en diversos países como Argentina, Brasil, China, Corea del Norte, Corea del Sur y Estados Unidos. Brasil representa aproximadamente el $27 \%$ de la oferta mundial de soya, ocupando el segundo lugar como mayor productor mundial de este grano, siendo superado sólo por Estados Unidos (Figueiredo et al., 2005).

Los contenidos de aceite y proteína de las semillas de soya son influenciados por efectos genéticos y ambientales. El efecto ambiental sobre tales características puede ser un factor importante durante el período de desarrollo de los granos. Esta influencia ambiental carece de estudios más detallados o precisos, debido probablemente a la existencia de varias interacciones (Pípolo, 2002). Los cambios en las concentraciones de nitrógeno disponible para las semillas, inducidas por el ambiente, pueden ser el mecanismo que explica las variaciones en la concentración de nitrógeno de las semillas cuando crecen en condiciones de campo (Hayati et al., 1996).

Después de la maduración fisiológica de la planta de soya, puede considerarse a la semilla como almacenada en campo, siempre y cuando la cosecha no se realice (Costa et al., 1983). El retraso de la cosecha de soya después de este período, constituye una de las principales causas de la disminución del poder germinativo y del vigor de las semillas. Esta reducción es determinada por factores genéticos, además de las condiciones ambientales a que están expuestas las semillas.

La semilla es uno de los principales insumos para la agricultura, siendo su calidad un factor determinante del éxito o el fracaso de la empresa agrícola (Johnston et al., 2003; Gaviola et al., 2006). En los últimos años, los agricultores están exigiendo mayor calidad de los insumos, y en este contexto la calidad de las semillas representa, actualmente, el blanco más frecuente de exigencias. La alta calidad de las semillas es un factor clave de los sistemas agrícolas de producción de granos. La calidad es necesaria para asegurar una adecuada población de plantas, una tasa de germinación razonable, y en diversas condiciones ambientales. La calidad de las semillas en la plantación representa los efectos integrados del ambiente durante la producción de semillas y las condiciones en las que las semillas se expusieron durante la cosecha y el almacenamiento (Egli et al., 2005).

Según Vieira et al. (2001) todo programa de control de calidad en la producción de semillas de un determinado cultivo, debe incluir el vigor como una característica principal, que debe ser evaluada en condiciones de laboratorio, debido a que este parámetro comprende las propiedades que determinan el potencial para un crecimiento rápido y uniforme, y el desarrollo de plantas consideradas normales bajo un amplio rango de condiciones de climas y suelos. El uso de semillas de baja calidad, sumado a la presencia de condiciones ambientales adversas, pueden resultar en un bajo porcentaje de germinación.

El objetivo del presente trabajo fue evaluar los contenidos de aceite y de proteínas en las semillas de soya, contenido de proteína en el afrecho, y características fisiológicas de las semillas de cuatro cultivares de soya, evaluadas en distintos períodos de cosecha y cultivadas en dos ambientes del Estado de Mato Grosso do Sul, Brasil.

\section{MATERIALES Y MÉTODOS}

Se establecieron dos ensayos de campo con cuatro genotipos de soya en la temporada agrícola 20042005 , en campos experimentales pertenecientes a productores privados de la Hacienda Recanto ( $21^{\circ} 00^{\prime}$ lat. Sur; $55^{\circ} 01^{\prime}$ long. Oeste; 464 m.s.n.m.) y la empresa Sementes Guerra S.A. ( $22^{\circ} 11^{\prime}$ lat. Sur; 54 $55^{\prime}$ ' long. Oeste; 464 m.s.n.m.), ubicados en los Municipios de Sidrolândia y Dourados, respectivamente, del Estado de Mato Grosso do Sul, Brasil. Según la clasificación de Köppen (Feddema, 2005), Sidrolândia posee un clima definido como “Aw", mega-térmico, con invierno predominantemente seco, y Dourados tiene un clima definido como "Cwa", meso-térmico húmedo, con verano lluvioso.

Los genotipos de soya fueron: CD 202 (caracterizada por ciclo precoz), BRS 239 (ciclo precoz), BRS 133 (ciclo semi-precoz) y BRS 206 (ciclo pre$\mathrm{coz}$ ). Cultivares pertenecientes al grupo de maduración precoz, son aquellos que poseen un ciclo (de cosecha) inferior a 115 días (EMBRAPA, 2007). 
La siembra de soya se realizó en el segundo decenio de noviembre, que es considerado una época preferencial para las condiciones locales.

El diseño experimental fue de bloques completos al azar, con tres repeticiones y tratamientos en parcelas subdivididas. Las parcelas estaban constituidas por los cuatro cultivares de soya, establecidos en los dos sitios (Dourados y Sidrolândia). Las subparcelas correspondieron a cuatro épocas de cosecha: $\mathrm{R}_{7}, 7,14$ y 21 días después de la primera época. El estado de desarrollo $\mathrm{R}_{7}$ es el inicio del proceso de madurez de la soya (punto de madurez fisiológica), la cual ocurre cuando las plantas presentan una vaina con coloración amarillenta, que es el aspecto típico de una vaina madura. El punto de madurez fisiológica ocurre cuando las semillas alcanzan la máxima acumulación de materia seca y calidad fisiológica, la cual es evaluada por medio de la germinación y el vigor (Elmore et al., 2001).

El modelo estadístico considerado fue el siguiente:

$$
\begin{aligned}
y_{i j k l}= & \mu+S_{i}+C_{j}+(S x C)_{i j}+B(S)_{k(i)}+\delta_{j k}+E_{l}+ \\
& (C x E)_{j l}+(S x E)_{i l}+(S x C x E)_{i j l}+\varepsilon_{i j k l}
\end{aligned}
$$

donde $y$ representa el valor observado; $\mu$ es la media general del experimento; $S$ representa el efecto del sitio o localidad; $C$ es el efecto del cultivar o genotipo; $(S x C)$ es el efecto de la interacción sitiocultivar; $B(S)$ es el efecto del bloque; $\delta$ es el efecto residual de las parcelas; $E$ es el efecto de la época; (CxE) simboliza el efecto de la interacción cultivar-época; $(S x E)$ es el efecto de la interacción sitio-época; $(S x C x E)$ simboliza el efecto de la interacción sitio-cultivar-época; y $\varepsilon$ es el efecto residual de las subparcelas.

Las parcelas de cultivares de soya se sembraron en ocho hileras de $10 \mathrm{~m}$ de largo, espaciadas a $0,45 \mathrm{~m}$. El área útil de las unidades experimentales fue de $4,5 \mathrm{~m}^{2}$. La preparación de suelo, la fertilización y las demás prácticas culturales fueron las mismas sugeridas por el sistema de producción de la región (EMBRAPA, 2004). Se analizó el suelo donde los ensayos experimentales se establecieron en función de las características químicas, y consecuentemente la fertilización se realizó de acuerdo con las recomendaciones de EMBRAPA (2004).

Para asegurar el número suficiente de plantas a ser analizadas, durante la siembra se consideraron mayores cantidades de semillas que las indicadas para los ensayos. Dos semanas después de la emergencia de las plantas, se dejaron aproximadamente 14 plantas por metro lineal de soya (densidad de 311.111 plantas ha $^{-1}$ ). Después de la siembra de soya se consideró riego por aspersión para garantizar el establecimiento inicial de las plantas. En la siembra, las semillas de soya se trataron con fungicida y se inocularon con inoculante a base de Bradyrhizobium japonicum.

Las malezas se controlaron por aplicación de herbicidas y manualmente. Cada vez que fue necesario se realizó control de las plagas, a través de pulverizaciones sistemáticas de insecticidas recomendados para cada especie de insecto.

La cosecha se realizó manualmente en las cuatro épocas. Después de la cosecha, las semillas de soya se sacaron de las vainas, se colocaron en bolsas de papel kraft, y se mantuvieron en cámara fría, a 10 ${ }^{\circ} \mathrm{C}$ y con $50 \%$ de humedad relativa, hasta el final de la última cosecha e inicio de las evaluaciones de laboratorio.

El análisis químico de determinación de proteína bruta se realizó en el laboratorio de análisis de suelos de EMBRAPA Agropecuaria Oeste, institución perteneciente al Ministerio de Agricultura de Brasil, localizada en Dourados, Estado de Mato Grosso do Sul. Los análisis estándares de germinación, envejecimiento acelerado y emergencia de las plantas en substrato de arena se realizaron en el laboratorio NUPAGRI (Núcleo de Investigaciones Aplicadas a la Agricultura), perteneciente a la Universidad Estatal de Maringá, ubicada en Maringá, Estado de Paraná, Brasil.

Para cada característica se consideraron las siguientes referencias metodológicas: Análisis de germinación: se realizó de acuerdo a las instrucciones contenidas en las Reglas para el Análisis de Semillas del Ministerio de Agricultura de Brasil (Ministério da Agricultura, 1992); Envejecimiento acelerado: se realizó conforme a la metodología descrita por Krzyzanowski et al. (1991) y Marcos-Filho (1999). Este análisis es utilizado para evaluar el vigor de las semillas, y consiste en colocar las semillas en cajas plásticas, sobre telas de acero inoxidable que contienen $40 \mathrm{~mL}$ de agua destilada, para mantener la humedad relativa cercana al $100 \%$, y colocarlas en una cámara incubadora regulada a una tempera- 
tura de $41{ }^{\circ} \mathrm{C}$, por un período de $48 \mathrm{~h}$. En esta condición las semillas son sometidas a un estrés o envejecimiento artificial, simulando un almacenamiento convencional por un período prolongado de tiempo; Emergencia de las plantas en substrato de arena: se realizó de acuerdo a las instrucciones contenidas en las Reglas para el Análisis de Semillas del Ministerio de Agricultura de Brasil (Ministério da Agricultura, 1992); Determinación de proteínas: se utilizó el método Kjeldahl, para la cuantificación de nitrógeno total, de acuerdo con AOAC (1975); Determinación de proteína esperada en el afrecho de soya: se determinó de acuerdo con Pípolo (2002). En este caso se utilizó el término proteína esperada porque el contenido de proteínas es evaluado indirectamente por el contenido de nitrógeno en los granos, utilizándose un factor de corrección. Para el cálculo de la conversión de nitrógeno en proteínas se utilizó un factor de corrección de 6,25 , y el porcentaje de proteínas se obtuvo basándose en la materia seca; Determinación de aceite: se utilizó la metodología de AOAC (1975) con modificaciones.

En el análisis de germinación, envejecimiento acelerado y emergencia de las plantas en substrato de arena, la evaluación se realizó considerando el número de semillas germinadas y el número de plántulas normales. Para el análisis estadístico estos datos se expresaron como porcentaje, teniendo en consideración las pruebas de estabilidad de las varianzas y la distribución normal de los datos, usando PROC MIXED de SAS (SAS Institute, 1996). Se consideraron normales aquellas plantas germinadas que no presentaron daños evidentes o cualquier otro tipo de respuesta especificada en las reglas para el análisis de semillas (Ministério da Agricultura, 1992).

Posteriormente, los datos colectados se sometieron a análisis de varianza considerando ambos sitios, en esquema de parcelas subdivididas. Los valores medios de cultivares de soya se compararon a través del estadístico Tukey ( $5 \%$ de probabilidad). Se realizaron análisis de regresión para verificar el comportamiento de las características en función de las épocas de cosecha, para cada genotipo, considerando un nivel de $5 \%$ de probabilidad.

Se verificó el comportamiento de la distribución de los residuos a través del estadístico de normalidad de Shapiro y Wilk (1965), además de gráficos de frecuencias observadas en los análisis individuales por sitio. Para ello se utilizó el procedimiento PROC UNIVARIATE, con la opción NORMALTEST, de SAS Institute (SAS, 1996).

\section{RESULTADOS Y DISCUSIÓN}

El análisis de varianza considerando los sitios en conjunto reveló diferencias estadísticas significativas $(\mathrm{p}<0,05)$ para los efectos del sitio para la característica envejecimiento acelerado; cultivar, para las variables germinación, proteínas, aceite y proteína en el afrecho de soya; y épocas de cosecha, para germinación, envejecimiento acelerado, y emergencia de las plantas. Diferencias significativas fueron también evidenciadas en los efectos de interacción sitio-cultivar, para las variables germinación y emergencia de las plantas; e interacción sitio-época de cosecha, para la variable emergencia de las plántulas (Cuadro 1).

Al comparar los resultados del análisis estándar de germinación y el análisis de emergencia de las plántulas en substrato de arena, dentro de cada sitio (Cuadro 2), se observó que los cvs. BRS 133 y BRS 239 presentaron los mayores porcentajes de germinación, en Sidrolândia y Dourados, respectivamente; sin embargo, en este último sitio, el cv. CD 202 no fue estadísticamente diferente del cv. BRS 239, en el sitio de Dourados. Los cvs. BRS 206 y CD 202 presentaron el menor porcentaje de germinación y emergencia de plántulas en el sitio de Sidrolândia, y en el sitio de Dourados CD 202 presentó el valor más bajo en ambas características.

En el análisis entre sitios, los cvs. BRS 239 y CD 202 evidenciaron los mayores porcentajes de germinación y vigor en la región de Dourados, y los cvs. BRS 133 y BRS 206 no presentaron diferencias estadísticas para ambos análisis entre ambos sitios.

Se comprobó que los cvs. BRS 239 y CD 202 son relativamente aptos para la producción de semillas en el sitio de Dourados, bajo las condiciones establecidas en el ensayo. Esto considerando un $80 \%$ como el valor mínimo de germinación aceptable para la siembra de soya en la región y un establecimiento adecuado de las plantas en el campo (EMBRAPA, 1993). Aunque los valores encontrados están próximos al valor mínimo esperado, son estadísticamente diferentes a los cvs. BRS 133 y BRS 206. 
Cuadro 1. Análisis de varianza de las características germinación (GER), envejecimiento acelerado (ENV), emergencia de las plántulas en substrato de arena (EA), determinación de proteínas en las semillas (PROT), determinación de aceite (AC), y proteína esperada en el afrecho (PE), en función de las semillas de cuatro cultivares de soya, cuatro épocas de colecta, y dos sitios del Estado de Mato Grosso do Sul, Brasil.

Table 1. Analysis of variance for germination (GER), accelerated aging (ENV), seedling emergence on sand substrate (EA), protein content in seeds (PROT), oil content (AC) and protein content in soybean meal (PE), in function of four cultivars, four harvest periods and two sites of the State of Mato Grosso do Sul, Brazil.

\begin{tabular}{|c|c|c|c|c|c|c|c|}
\hline \multirow[t]{3}{*}{ Fuentes de variación } & \multirow[t]{3}{*}{ GL } & \multicolumn{6}{|c|}{ Cuadrados medios } \\
\hline & & GER & ENV & EA & PROT & AC & PE \\
\hline & & \multicolumn{6}{|c|}{$(\%)$} \\
\hline Sitio $(S)$ & 1 & $1.014,0 \mathrm{~ns}$ & $720,5 *$ & $981,8 \mathrm{~ns}$ & $14,3 \mathrm{~ns}$ & $1,5 \mathrm{~ns}$ & $2,3 \mathrm{~ns}$ \\
\hline Cultivar (C) & 3 & $253,9 *$ & $107,0 \mathrm{~ns}$ & $230,1 \mathrm{~ns}$ & $125,9 *$ & $64,1 *$ & $144,7 *$ \\
\hline $\mathrm{S} \times \mathrm{C}$ & 3 & $278,8 *$ & $66,8 \mathrm{~ns}$ & $271,7 *$ & $6,7 \mathrm{~ns}$ & $0,4 \mathrm{~ns}$ & $0,1 \mathrm{~ns}$ \\
\hline Bloque/S & 4 & 69,4 & 119,0 & 52,4 & 15,6 & 1,3 & 3,8 \\
\hline Error a & 12 & 24,2 & 33,9 & 18,1 & 16,6 & 1,4 & 2,6 \\
\hline Época (E) & 3 & $443,4 *$ & $424,2 *$ & $443,8 *$ & $17,7 \mathrm{~ns}$ & $0,8 \mathrm{~ns}$ & $1,2 \mathrm{~ns}$ \\
\hline $\mathrm{C} \times \mathrm{E}$ & 9 & $6,1 \mathrm{~ns}$ & $16,1 \mathrm{~ns}$ & $12,5 \mathrm{~ns}$ & $11,2 \mathrm{~ns}$ & $0,5 \mathrm{~ns}$ & $1,8 \mathrm{~ns}$ \\
\hline $\mathrm{S} \times \mathrm{E}$ & 3 & $43,9 \mathrm{~ns}$ & $5,1 \mathrm{~ns}$ & $40,3 \mathrm{~ns}$ & $13,0 \mathrm{~ns}$ & $0,3 \mathrm{~ns}$ & $7,9 \mathrm{~ns}$ \\
\hline $\mathrm{S} \times \mathrm{C} \times \mathrm{E}$ & 9 & $11,2 \mathrm{~ns}$ & $35,0 \mathrm{~ns}$ & $9,2 \mathrm{~ns}$ & $19,6 \mathrm{~ns}$ & $0,6 \mathrm{~ns}$ & $8,4 \mathrm{~ns}$ \\
\hline Error b & 48 & 6,5 & 16,4 & 5,3 & 17,7 & 0,8 & 6,3 \\
\hline Media & & 75,0 & 69,0 & 75,4 & 33,5 & 20,4 & 43,4 \\
\hline $\mathrm{CV}_{1}, \%$ & & 6,6 & 8,4 & 5,7 & 12,2 & 5,7 & 3,7 \\
\hline $\mathrm{CV}_{2}, \%$ & & 3,4 & 5,9 & 3,1 & 12,6 & 4,5 & 5,8 \\
\hline
\end{tabular}

* Significativo a $5 \%$ de probabilidad, por la prueba F; ns: no significativo.

GL: grados de libertad; $\mathrm{CV}_{1}$ y $\mathrm{CV}_{2}$ : coeficientes de variación para los errores a y b, respectivamente.

Cuadro 2. Valores medios estimados en el análisis de germinación (GER) y emergencia de las plántulas en substrato de arena (EA), por cultivar de soya y sitio.

Table 2. Mean values estimates in the germination test (GER) and seedling emergence on sand substrates (EA), per soybean cultivar and site.

\begin{tabular}{llcl}
\hline Característica & Cultivar $^{1}$ & \multicolumn{2}{c}{ Sitio $^{2}$} \\
\cline { 3 - 4 } & & Sidrolândia & Dourados \\
\hline GER, \% & BRS 133 & $78,16 \mathrm{aA}$ & $76,83 \mathrm{bA}$ \\
& BRS 206 & $69,41 \mathrm{cA}$ & $72,33 \mathrm{cA}$ \\
& BRS 239 & $71,66 \mathrm{bB}$ & $83,83 \mathrm{aA}$ \\
& CD 202 & $67,91 \mathrm{cB}$ & $80,16 \mathrm{aA}$ \\
\cline { 2 - 4 } & Media & 71,79 & 78,29 \\
\hline EA, \% & BRS 133 & $78,75 \mathrm{aA}$ & $77,16 \mathrm{bA}$ \\
& BRS 206 & $69,83 \mathrm{cA}$ & $73,08 \mathrm{cA}$ \\
& BRS 239 & $71,58 \mathrm{bB}$ & $84,00 \mathrm{aA}$ \\
& CD 202 & $68,66 \mathrm{cB}$ & $80,16 \mathrm{aA}$ \\
\hline & Media & 72,21 & 78,60 \\
\hline
\end{tabular}

${ }^{1}$ Medias seguidas de la misma letra minúscula en la columna, no difieren entre sí por el test de Tukey a 5\% de probabilidad. ${ }^{2}$ Medias seguidas de la misma letra mayúscula, en la línea, no difieren entre sí por el test de F a 5\% de probabilidad. 
El análisis de emergencia de plántulas en substrato de arena permite determinar el vigor relativo del lote de semillas, es decir, las semillas poco vigorosas de un determinado cultivar serían incapaces de germinar y producir plántulas consideradas normales en un suelo y ambiente climático desfavorable a la germinación. Los cvs. BRS 133, en Sidrolândia, y BRS 239 y CD 202, en Dourados, presentaron una menor susceptibilidad al deterioro en campo y, por lo tanto, mejor calidad relativa de las semillas. La reducción del vigor de las semillas está relacionada al proceso de deterioro causado por varios factores, entre otros: cosechas tardías, lluvias, y secado y/o almacenamiento inadecuados. Uno de los síntomas del deterioro de las semillas es la disminución de la resistencia al estrés ambiental durante la emergencia y crecimiento inicial de las plantas. Las semillas deterioradas poseen baja germinación y vigor, y por consiguiente tienden a producir plántulas débiles con reducido potencial de rendimiento. Según Egli et al. (2005) las condiciones ambientales desfavorables (del punto de vista de la temperatura, lluvia o humedad relativa) durante el crecimiento y desarrollo de la semilla en el campo, pueden reducir la germinación y el vigor de las semillas de soya.

En el análisis del efecto principal del cultivar (Cuadro 3), considerando los dos ambientes, los genotipos BRS 133 y BRS 239 presentaron los mayores porcentajes de germinación, y el cv. BRS 206 evidenció la respuesta menor. Sin embargo, todos los cultivares presentaron bajos porcentaje de germinación, debido, probablemente, a la presencia de temperaturas elevadas y la falta de lluvia durante el período de desarrollo de los granos, que también obligó a realizar riegos por aspersión para suplir las necesidades mínimas de agua. Algunas investigaciones indican que el estrés por sequía durante el desarrollo de la semilla también reduce la calidad de la semilla (Smicklas et al., 1992; Heatherly, 1993; Egli et al., 2005). Adicionalmente, Egli et al. (2005) mencionan que las condiciones secas durante la época de cosecha pueden aumentar la lesión física y reducir la calidad de las semillas si éstas se manejan en niveles de baja humedad.

Los cvs. BRS 133 y BRS 206 presentaron los mayores contenidos de proteínas en la semilla de soya, y a su vez, el cv. CD 202 presentó el menor valor para esta característica (Cuadro 3). En la determinación de aceite, los cultivares mostraron un efecto relativo inverso en relación con el contenido de proteína, en el cual el cv. CD 202 evidenció los mayores valores de porcentaje de aceite, difiriendo estadísticamente de los otros cultivares. Los cvs. BRS 133 y BRS 206 no presentaron diferencias significativas entre sí, $\mathrm{y}$ evidenciaron los menores contenidos de aceite.

Los contenidos de proteínas y aceite fueron relativamente bajos debido a la mala distribución de las lluvias durante el desarrollo de los granos, en ambos sitios de estudio. Este factor, en conjunto con la disponibilidad de nitrógeno para las semillas, son piezas claves que pueden provocar variaciones en los contenidos de proteína y aceite de las semillas de soya. Entre los factores climáticos, la temperatura es también citada como un factor que podría explicar las diferencias de concentraciones de proteínas y aceite en la semilla de soya (Pípolo, 2002).

Cuadro 3. Valores medios estimados en el análisis de germinación (GER), determinación de proteína (PROT), determinación de aceite (AC), y proteína esperada en el afrecho de soya (PE), por cultivar de soya. Temporada agrícola 2004-2005.

Table 3. Mean value estimates for germination (GER), protein content (PROT), oil content (AC) and expected protein in soybean bran (PE), per soybean cultivar. 2004-2005 growing season.

\begin{tabular}{lllll}
\hline \multirow{2}{*}{ Cultivar } & \multicolumn{5}{c}{ Característica } \\
\cline { 2 - 5 } & GER & PROT & AC & PE \\
\cline { 2 - 5 } & & & $(\%)$ \\
\hline BRS 133 & $77,31 \mathrm{a}$ & $35,00 \mathrm{a}$ & $19,83 \mathrm{c}$ & $43,04 \mathrm{a}$ \\
BRS 206 & $70,65 \mathrm{c}$ & $35,67 \mathrm{a}$ & $18,75 \mathrm{c}$ & $46,83 \mathrm{a}$ \\
BRS 239 & $77,48 \mathrm{a}$ & $32,63 \mathrm{~b}$ & $20,54 \mathrm{~b}$ & $42,50 \mathrm{~b}$ \\
CD 202 & $73,71 \mathrm{~b}$ & $30,67 \mathrm{c}$ & $22,63 \mathrm{a}$ & $41,08 \mathrm{c}$ \\
Media & 74,79 & 33,49 & 20,44 & 43,36 \\
\hline
\end{tabular}

Medias con la misma letra en cada columna no difieren entre sí, por el test de Tukey a 5\% de probabilidad. 
Los resultados de proteína esperada en el afrecho de soya están relativamente correlacionados con la determinación de proteínas, para la cual los cvs. BRS 133 y BRS 206 no evidenciaron diferencias significativas entre sí (Cuadro 3) y mostraron el mayor porcentaje de proteínas en el afrecho. El cv. CD 202 presentó el menor porcentaje de contenido proteico. En los niveles de proteína esperada en el afrecho de soya, un resultado importante fue que solamente el cv. BRS 206 alcanzó el mínimo de $46 \%$ exigido para la exportación de alta calidad. Estos resultados refuerzan la necesidad de atención para el factor proteína de los granos en los programas de mejoramiento de la especie. En el caso del cv. BRS 206, se trata de un genotipo adaptado a la región, con rendimiento aceptable, y con buena estabilidad en la característica de proteína de los granos.

La ecuación de regresión que mejor se ajustó en el análisis de germinación de las semillas (Figura 1) correspondió a un modelo cuadrático, el cual presentó un punto máximo de 8 días de cosecha, y respuesta máxima de $78,58 \%$ de plántulas normales. En las épocas de cosechas realizadas con 14 y 21 días, el porcentaje de germinación comienza a disminuir, no satisfaciendo los niveles de calidad necesarios para la producción de semillas. La utilización de semillas de alta calidad permitiría garantizar el éxito de las siembras de soya, y con ello se aumentaría la productividad agrícola, se maximizaría el aprovechamiento de los demás insumos aplicados en el suelo, se evitaría la diseminación

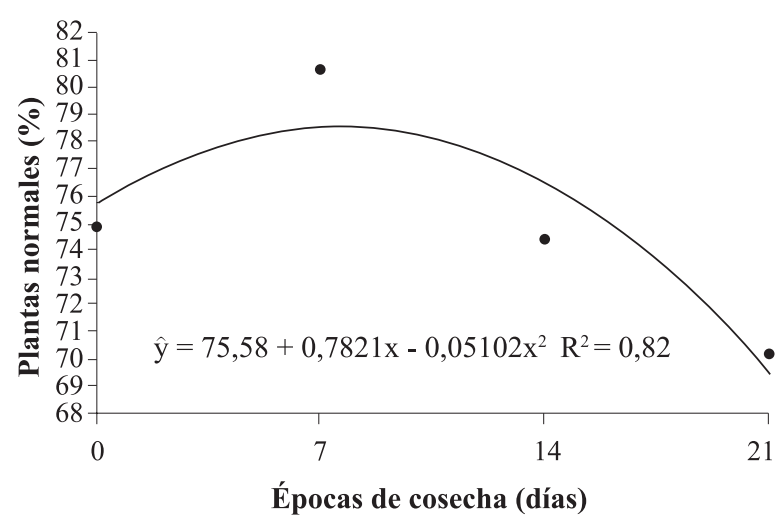

Figura 1. Plántulas normales en función de las épocas de cosecha, para el análisis estándar de germinación.

Figure 1. Normal seedlings versus harvest periods, for the germination test. de enfermedades y se reducirían las pérdidas y el riesgo en la cosecha (Hamman et al., 2002).

En el análisis de envejecimiento acelerado, la ecuación de regresión que mejor se ajustó en el porcentaje de plántulas normales versus épocas de cosecha fue el modelo cuadrático (Figura 2), evidenciando un punto máximo de 7 días de cosecha y respuesta máxima de $72,38 \%$ de plántulas normales. A partir de este punto los niveles de vigor comienzan a disminuir, ya que las semillas de soya serían consideradas como almacenadas en campo, y por lo tanto, sujetas a las variaciones de precipitación y temperatura. Tales condiciones ejercen un papel fundamental en la calidad fisiológica de las semillas, y reducen, en algunas situaciones, su calidad comercial. Según Marcos-Filho et al. (1987), el principio del método de envejecimiento acelerado se basa en el hecho que las semillas con un alto vigor mantendrían su viabilidad cuando son sometidas durante ciertos períodos a condiciones severas de temperatura y humedad relativa, mientras que las semillas de bajo vigor reducirían su viabilidad en las mismas condiciones.

Para la característica porcentaje de plántulas normales obtenidas en el análisis de emergencia en substrato de arena, la ecuación de regresión que mejor se ajustó fue el modelo cuadrático, estimando un punto de máximo con 7 días de cosecha y respuesta máxima de $78,74 \%$ de plántulas normales (Figura 3). Cosechas realizadas con 14 y 21 días muestran nuevamente una caída acentuada en el

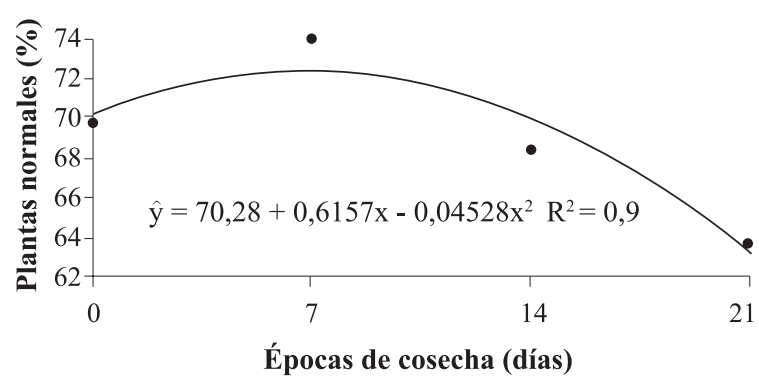

Figura 2. Plántulas normales en función de cuatro épocas de cosecha, para las pruebas de envejecimiento acelerado.

Figure 2. Normal seedlings versus harvest periods, for accelerated aging. 


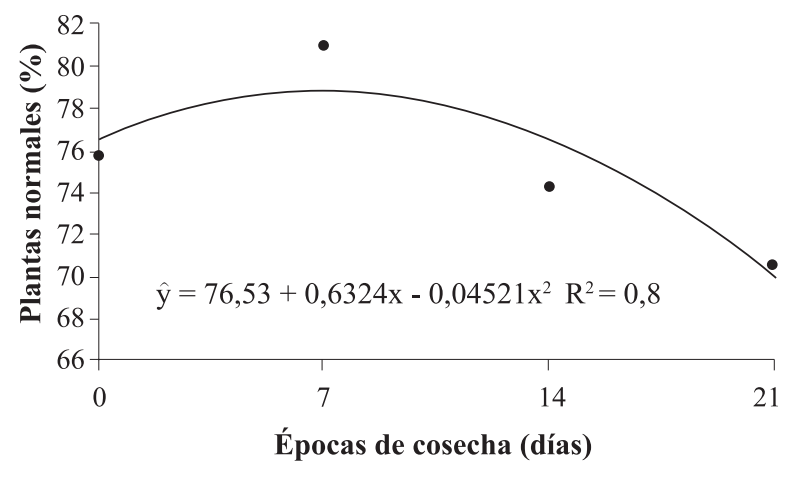

Figura 3. Plántulas normales en función de las cuatro épocas de cosecha, para el análisis de emergencia de las semillas de soya en substrato de arena.

Figure 3. Normal seedlings versus harvest periods, for the analysis of seedling emergence on sand substrate.

vigor, indicando que cuando los cultivares son cosechados en esas dos épocas, las semillas son menos vigorosas y generalmente son más afectadas en su capacidad de producir plántulas normales. Estos resultados son coincidentes con lo encontrado por Nakagawa et al. (1985), quienes verificaron que el efecto de la calidad de semillas de soya fue significativo durante la emergencia de plántulas, con vigor reducido debido al retardo de la cosecha, afectando la emergencia en campo.

El uso de semillas de baja calidad en las siembras de soya, sumado a condiciones ambientales adver- sas, pueden resultar en un bajo porcentaje de germinación y menor velocidad de emergencia de las plantas. Por otro lado, las semillas consideradas de alto vigor, normalmente presentan una germinación más rápida y uniforme, siendo capaces de soportar mejor las adversidades del ambiente (Lopes et al., 2002). Hamman et al. (2002), en un estudio relacionado con las características fisiológicas de las semillas de soya, confirmaron las ventajas de las semillas con alto vigor en ambientes considerados adversos para su desarrollo.

\section{CONCLUSIONES}

Los contenidos de proteínas de las semillas, afrecho y aceite, fueron dependientes sólo del genotipo estudiado. Los cvs. BRS 133 y BRS 206 mostraron los mayores contenidos de proteína, en tanto que para aceite estos cultivares fueron los de menor valor, siendo superados significativamente por el cv. CD 202.

Interacciones del genotipo con el ambiente fueron importantes sólo para las características de germinación y envejecimiento acelerado.

A medida que se atrasó la época de cosecha, se observó una reducción significativa en el potencial germinativo y en el vigor de las semillas, características que podrían determinar el potencial para un crecimiento rápido y adecuado desarrollo de la planta de soya.

\section{LITERATURA CITADA}

AOAC. 1975. Official methods of analysis. $1054 \mathrm{p}$. Association of Official Analytical Chemists (AOAC), Washington D.C., USA.

Costa, N.P, J.B. França-Neto, L.A.G. Pereira, A.A. Henning, L. Turkienicz, e M.C. Dias. 1983. Antecipação de colheitas de sementes de soja através do uso de dessecantes. Revista Brasileira de Sementes 3:183-198.

Egli, D.B., D.M. TeKrony, J.J. Heitholt, and J. Rupe. 2005. Air temperature during seed filling and soybean seed germination and vigor. Crop Sci. 45:1329-1335.

Elmore, R.W., F.W. Roeth, R.N. Klein, S.Z. Knezevic, A. Martin, L.A. Nelson, and C.A. Shapiro. 2001. Glyphosate-resistant soybean cultivar response to glyphosate. Agron. J. 93:404-407.
EMBRAPA. 1993. Serviço de produção de sementes básicas. Padrões estaduais de sementes. p. 35-37. Empresa Brasileira de Pesquisa Agropecuaria (EMBRAPA), Brasília D.F., Brasil.

EMBRAPA. 2004. Tecnologias de produção de soja: Região Central do Brasil. 239 p. Empresa Brasileira de Pesquisa Agropecuaria (EMBRAPA), Londrina, Brasil.

EMBRAPA. 2007. Tecnologias de produção de soja Paraná 2007. 217 p. Empresa Brasileira de Pesquisa Agropecuaria (EMBRAPA), Londrina, Brasil.

Feddema, J.J. 2005. A revised thornthwaite-type global climate classification. Physical Geography 26(6):442466. 
Figueiredo, M.G., A.L.M. Barros, e J.J.M. Guilhoto. 2005. Relação econômica dos setores agrícolas do Estado do Mato Grosso com os demais setores pertencentes tanto ao Estado quanto ao restante do Brasil. Revista de Economia e Sociologia Rural 43(3):557-575.

Gaviola, J., A. Ordovini, R. Lepez, y M.A. Makuch. 2006. Evolución de la calidad de semillas de cebolla almacenadas en condiciones no controladas. Agric. Téc. (Chile) 66:13-20.

Hamman, B., D.B. Egli, and G. Koning. 2002. Seed vigor, soilborne pathogens, preemergent growth, and soybean seedling emergence. Crop Sci. 42:451-457.

Hayati, R., D.B. Egli, and S.J. Crafts-Brandner. 1996. Independence of nitrogen supply and seed growth in soybean: studies using in vitro culture system. J. Exp. Bot. 47:33-44.

Heatherly, L.G. 1993. Drought stress and irrigation effects on germination of harvested soybean seed. Crop Sci. 33:777-781.

Johnston, M.R., A. Olivares, y J. Laura. 2003. Producción y calidad de semillas en cuatro Poaceas. Efecto de cortes con distintas frecuencias y en diversas etapas fenológicas. Agric. Téc. (Chile) 63:146-155.

Krzyzanowski, F.C., J.B. França-Neto, e A.A. Henning. 1991. Relato dos testes e vigor disponíveis para grandes culturas. p. 15-50. Associação Brasileira de Tecnologia de Sementes, Londrina, Brasil.

Lopes, J.C., S. Martins-Filho, C. Tagliaferri, e O.J.P. Rangel. 2002. Avaliação da qualidade fisiológica de sementes de soja produzidas em Alegre-ES. Revista Brasileira de Sementes 24:51-58.

Marcos-Filho, J. 1999. Teste de envelhecimento acelerado. p. 1-24. In Krzyzanowski, F.C., R.D. Vieira e J.B. França-Neto (eds.). Vigor de sementes: conceitos e testes. Associação Brasileira de Tecnologia de Sementes, Londrina, Brasil.
Marcos-Filho, J., S.M. Cicero, e W.R. Silva. 1987. Avaliação da qualidade das sementes. 230 p. Fundação de Estudos Agrários Luiz de Queiroz, Piracicaba, São Paulo, Brasil.

Ministério da Agricultura. 1992. Regras para análise de sementes. 365 p. Ministério da Agricultura do Abastecimento e da Reforma Agrária, Brasília DF., Brasil.

Nakagawa, J., J.R. Machado, e C.A. Rosolem. 1985. Efeito da qualidade da semente sobre o estabelecimento da população e outras características da soja. Revista Brasileira de Sementes 7(2):47-62.

Pípolo, A.E. 2002. Influência da temperatura sobre as concentrações de proteína e óleo em sementes de soja (Glycine max (L.) Merrill). 128 p. Tesis de Doctorado. Universidade de São Paulo, Escola Superior de Agricultura Luiz de Queiroz. Piracicaba, São Paulo, Brasil.

SAS. 1996. Statistical analysis system. User's guide. 956 p. SAS Institute, Cary, North Carolina, USA.

Shapiro, S.S., and M.B. Wilk. 1965. An analysis of variance test for normality (complete samples). Biometrika 52:591-611.

Smicklas, K.D., R.E. Mullen, R.E. Carlson, and A.D. Knapp. 1992. Soybean seed quality response to drought stress and pod position. Agron. J. 84:166-170.

Vieira, R.D., S.R.M. Bittencourt, e M. Panobianco. 2001. Vigor: um componente da qualidade de sementes. 199 p. Associação Brasileira de Tecnologia de Sementes, Londrina, Brasil. 